IC $/ 95 / 50$

hep-ph/9505273

International Atomic Energy Agency

and

United Nations Educational Scientific and Cultural Organization

INTERNATIONAL CENTRE FOR THEORETICAL PHYSICS

\title{
ON THE FORM FACTORS OF THE $D_{s}^{+} \rightarrow \phi \mu^{+} \nu_{\mu}$ DECAY
}

\author{
F. Hussain, A.N. Ivanov ${ }^{1}$, N.I. Troitskayaf \\ International Centre for Theoretical Physics, Trieste, Italy.
}

\begin{abstract}
We apply the infinite mass effective theory, when a heavy quark mass tends to infinity, and Chiral perturbation theory at the quark level, based on the extended Nambu - Jona - Lasinio model with linear realization of chiral $U(3) \times U(3)$ symmetry, to calculate the form factors of the $D_{s}^{+} \rightarrow \phi \mu^{+} \nu_{\mu}$ decay up to the first order in current $s$ - quark mass. The theoretical results are compared with experimental data and found to be in good agreement.

MIRAMARE - TRIESTE

May 1995

\footnotetext{
${ }^{1}$ Permanent Address: Department of Theoretical Physics, State Technical University, 195251 St. Petersburg, Russian Federation.
} 


\section{Introduction}

In our recent publications [1-3] we considered the form factors of the semileptonic $D \rightarrow \bar{K}^{*}(\bar{K}) e^{+} \nu_{e}$ decays both in the chiral limit $[1,2]$ and at first order in current $s$-quark mass expansion [3]. For the description of $D$-mesons we applied the infinite mass effective theory (IMET) $[4,5]$, when the $c$-quark mass $M_{c}$ tends to infinity, i.e. $M_{c} \rightarrow \infty$. In the IMET, we describe the long - distance physics within Chiral perturbation theory at the quark level $(\mathrm{CHPT})_{q}[6]$, based on the extended Nambu-Jona-Lasinio (ENJL) model with linear realization of chiral $U(3) \times U(3)$ symmetry [7]. The IMET supplemented by $(\mathrm{CHPT})_{q}$ has been successfully applied to the description of the fine structure of the mass spectra of non-strange $D\left(D^{*}\right)$ and strange $D_{s}^{+}\left(D_{s}^{*+}\right)$ and leptonic constants, caused by first order corrections in current-quark mass expansion [8]. The computation of the probabilities of strong and electromagnetic $D^{*}$ decays performed within IMET and $(\mathrm{CHPT})_{q}$ has given good results compared with experimental data [9].

In this paper we apply IMET and $(\mathrm{CHPT})_{q}$ to the calculation of the form factors of the $D_{s}^{+} \rightarrow \phi \mu^{+} \nu_{\mu}$ decay, keeping corrections up to first order in current $s$-quark mass.

\section{The form factors of the $D_{s}^{+} \rightarrow \phi \mu^{+} \nu_{\mu}$ decay}

The amplitude of the $D_{s}^{+} \rightarrow \phi \mu^{+} \nu_{\mu}$ decay is determined as follows

$M\left(D_{s}^{+} \rightarrow \phi \mu^{+} \nu_{\mu}\right)=-\frac{G_{F}}{\sqrt{2}} V_{c s}^{*}<\phi(Q)\left|\bar{s}(0) \gamma_{\alpha}\left(1-\gamma^{5}\right) c(0)\right| D_{s}^{+}(p)>\ell^{\alpha}$

where $G_{F}=1.166 \times 10^{-5} \mathrm{GeV}^{-2}$ is the Fermi weak coupling constant, $\left|V_{c s}\right|=0.975$ is the CKM mixing matrix element, $s(0)$ and $c(0)$ are the $s$ and $c$ current quark fields with $N$ colour degrees of freedom each, and $\ell^{\alpha}=\bar{u}\left(k_{\nu_{\mu}}\right) \gamma^{\alpha}\left(1-\gamma^{5}\right) v\left(k_{\mu^{+}}\right)$is the weak leptonic current.

The hadronic matrix element

$$
M_{\alpha}\left(D_{s}^{+} \rightarrow \phi\right)=<\phi(Q)\left|\bar{s}(0) \gamma_{\alpha}\left(1-\gamma^{5}\right) c(0)\right| D_{s}^{+}(p)>
$$

can be parametrized in terms of four form factors [3] 


$$
\begin{aligned}
M_{\alpha}\left(D_{s}^{+} \rightarrow \phi\right) & =i a_{1}\left(q^{2}\right) \epsilon_{\alpha}^{*}(Q)-i a_{2}\left(q^{2}\right)\left(\epsilon^{*}(Q) \cdot p\right)(p+Q)_{\alpha} \\
& -i a_{3}\left(q^{2}\right)\left(\epsilon^{*}(Q) \cdot p\right)(p-Q)_{\alpha} \\
& -2 b\left(q^{2}\right) \varepsilon_{\alpha \beta \mu \nu} \epsilon^{* \beta}(Q) p^{\mu} Q^{\nu}
\end{aligned}
$$

where $q^{2}$ is the square invariant mass of the lepton pair such that $m_{\mu}^{2} \leq$ $q^{2} \leq\left(M_{D_{s}^{+}}-M_{\phi}\right)^{2}$ where $M_{D_{s}^{+}}, M_{\phi}$ and $m_{\mu}$ are the masses of the $D_{s}^{+}, \phi$ and $\mu^{+}$mesons respectively and $\epsilon^{*}$ is the polarisation tensor of the outgoing $\phi$ meson.

We shall seek the form factors $a_{i}\left(q^{2}\right)(i=1,2,3)$ and $b\left(q^{2}\right)$ in the form of an expansion in powers of the current $s$-quark mass upto first order terms

$$
\begin{aligned}
a_{i}\left(q^{2}\right) & =a_{i}^{(0)}\left(q^{2}\right)+a_{i}^{(1)}\left(q^{2}\right) \\
b\left(q^{2}\right) & =b^{(0)}\left(q^{2}\right)+b^{(1)}\left(q^{2}\right)
\end{aligned}
$$

The form factors $a_{i}^{(0)}\left(q^{2}\right)(i=1,2,3)$ and $b^{(0)}\left(q^{2}\right)$ are determined in the

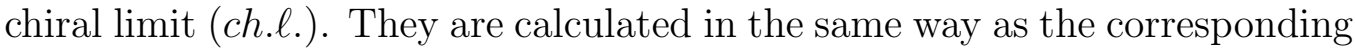
form factors for the process $D \rightarrow \bar{K}^{*} e^{+} \nu_{e}[1,3]$, that is

$$
\begin{aligned}
a_{1}^{(0)}\left(q^{2}\right) & =\sqrt{\frac{3}{8}} M_{*} \\
a_{2}^{(0)}\left(q^{2}\right) & =\sqrt{\frac{3}{8}} \frac{M_{*}}{M_{D}^{2}}\left[\frac{q^{2}}{M_{D}^{2}-q^{2}}\right. \\
& \left.+\frac{M_{D}^{2}-q^{2}}{M_{*}^{2}}\left(1-\frac{2 m M_{D}}{M_{D}^{2}-q^{2}}\right) \ln \left(1+\frac{M_{*}^{2}}{M_{D}^{2}-q^{2}}\right)\right] \\
a_{3}^{(0)}\left(q^{2}\right) & =-\sqrt{\frac{3}{8}} \frac{M_{*}}{M_{D}^{2}}\left[\frac{2 M_{D}^{2}-q^{2}}{M_{D}^{2}-q^{2}}\right. \\
& \left.-\frac{M_{D}^{2}-q^{2}}{M_{*}^{2}}\left(1-\frac{2 m M_{D}}{M_{D}^{2}-q^{2}}\right) \ln \left(1+\frac{M_{*}^{2}}{M_{D}^{2}-q^{2}}\right)\right] \\
b^{(0)}\left(q^{2}\right) & =\sqrt{\frac{3}{8}} \frac{1}{M_{*}} \ln \left(1+\frac{M_{*}^{2}}{M_{D}^{2}-q^{2}}\right) .
\end{aligned}
$$

Here we have denoted $M_{*}=\sqrt{M_{D} \bar{v}^{\prime} / 2}$ where $M_{D}=1.86 \mathrm{GeV}$ is the mass of the charmed pseudoscalar meson at the chiral limit and $\bar{v}^{\prime}=4 \Lambda=$ 
$2.66 \mathrm{GeV}$. The parameter $\Lambda$ appears as the cut-off in the Euclidian 3dimensional momentum space evaluation of constituent quark loop diagrams. This cut-off $\Lambda$ is connected with the scale of spontaneous breaking of chiral symmetry $(\mathrm{SBCS})$ in $(\mathrm{CHPT})_{q}$ via the relationship $\Lambda=\Lambda_{\chi} / \sqrt{2}=0.67 \mathrm{GeV}$ at $\Lambda_{\chi}=0.94 \mathrm{GeV}[6]$.

The form factors $a_{i}^{(1)}\left(q^{2}\right)(i=1,2,3)$ and $b^{(1)}\left(q^{2}\right)$ are determined by the matrix element [3]

$$
\begin{aligned}
& M_{\alpha}^{(1)}\left(D_{s}^{+} \rightarrow \phi\right)=-i m_{0 s} \\
& \times \int d^{4} x<h(Q)\left|\mathrm{T}\left(\bar{s}(x) s(x) \bar{s}(0) \gamma_{\alpha}\left(1-\gamma^{5}\right) c(0)\right)\right| D_{s}^{+}(p)>_{c h . \ell .} .
\end{aligned}
$$

In accordance with the procedure expounded in $[1-3,8,9]$ we can reduce the matrix element (6) to the expression

$$
\begin{aligned}
& M_{\alpha}^{(1)}\left(D_{s}^{+} \rightarrow \phi\right)=g_{D} m_{0 s} i, \int d^{4} x \int_{-\infty}^{\infty} d z_{0} \theta\left(-z_{0}\right) \times \\
& \times<\phi(Q)\left|\mathrm{T}\left(\bar{s}(x) s(x) \bar{s}(0) \gamma_{\alpha}\left(\frac{1+\gamma^{0}}{2}\right) \gamma^{5} s\left(z_{0}, \overrightarrow{0}\right)\right)\right| 0>_{c h . \ell .}
\end{aligned}
$$

obtained at leading order in the large $N$ and $M_{c}$ expansion. The coupling constant $g_{D}$ has been calculated in [9]

$$
g_{D}=\frac{2 \sqrt{2} \pi}{\sqrt{N}}\left(\frac{M_{D}^{2}}{M_{c} \bar{v}^{\prime}}\right)^{1 / 2}
$$

The r.h.s. of (17) involves only the light quark fields. Therefore for the evaluation of (7) we can apply $(\mathrm{CHPT})_{q}[1-3,6,8,9]$. Since the leading order of the r.h.s. of (7) in current quark mass expansion is fixed by the factor $m_{0 s}$, so the matrix element $\langle\phi(Q)|\mathrm{T}(\ldots)| 0>$ has to be calculated in the chiral limit.

In order to evaluate the matrix element (7) let us compare this with the matrix element $M_{\alpha}^{(1)}\left(D \rightarrow \bar{K}^{*}\right)$ describing the $D \rightarrow \bar{K}^{*}$ transition at the first order in current $s$ - quark mass expansion [3]

$$
M_{\alpha}^{(1)}\left(D \rightarrow \bar{K}^{*}\right)=g_{D} m_{0 s} i, \int d^{4} x \int_{-\infty}^{\infty} d z_{0} \theta\left(-z_{0}\right) \times
$$




$$
\times<\bar{K}^{*}(Q)\left|\mathrm{T}\left(\bar{s}(x) s(x) \bar{s}(0) \gamma_{\alpha}\left(\frac{1+\gamma^{0}}{2}\right) \gamma^{5} q\left(z_{0}, \overrightarrow{0}\right)\right)\right| 0>_{\text {ch.l. }}
$$

where $q=u$ or $d$ for $D^{0}$ or $D^{+}$, respectively.

By applying the formulas of quark conversion (Ivanov [6]) one can show that, between matrix elements $M_{\alpha}^{(1)}\left(D_{s}^{+} \rightarrow \phi\right)$ and $M_{\mu}^{(1)}\left(D \rightarrow \bar{K}^{*}\right)$, there is the relationship

$$
M_{\alpha}^{(1)}\left(D_{s}^{+} \rightarrow \phi\right)=2 M_{\alpha}^{(1)}\left(D \rightarrow \bar{K}^{*}\right)
$$

Readers can verify this relationship by noting that the $\phi$ meson possesses the quark structure $(\bar{s} s)$.

By virtue of the relationship (10) the form factors $a_{i}^{(1)}\left(q^{2}\right)(i=1,2,3)$ and $b^{(1)}\left(q^{2}\right)$ read [3]

$$
\begin{aligned}
a_{1}^{(1)}\left(q^{2}\right) & =\sqrt{3} \frac{m_{0 s}}{M_{*}} \frac{\bar{v}}{4 m} M_{D} \ln \left(\frac{\bar{v}^{\prime}}{4 m}\right) \\
a_{2}^{(1)}\left(q^{2}\right) & =-a_{3}^{(1)}\left(q^{2}\right)=b^{(1)}\left(q^{2}\right) \\
b^{(1)}\left(q^{2}\right) & =\sqrt{3} \frac{m_{0 s}}{M_{*}} \frac{\bar{v}}{4 m} \frac{M_{D}}{M_{D}^{2}-q^{2}}\left[1-\ln \left(1+\frac{M_{*}^{2}}{M_{D}^{2}-q^{2}}\right)\right] .
\end{aligned}
$$

Now we can get the numerical values of the form factors at $q^{2}=0$

$$
\begin{aligned}
& a_{1}(0)=a_{1}^{(0)}(0)+a_{1}^{(1)}(0)=0.96+0.28=1.24(\mathrm{GeV}), \\
& a_{2}(0)=a_{2}^{(0)}(0)+a_{2}^{(1)}(0)=0.14+0.06=0.20(\mathrm{GeV})^{-1}, \\
& a_{3}(0)=a_{3}^{(0)}(0)+a_{3}^{(1)}(0)=-0.42-0.06=-0.48(\mathrm{GeV})^{-1}, \\
& b(0)=b^{(0)}(0)+b^{(1)}(0)=0.21+0.06=0.27(\mathrm{GeV})^{-1} .
\end{aligned}
$$

One finds that the first order corrections in current $s$ - quark mass expansion are between 14 and $43 \%$. The form factors $a_{i}\left(q^{2}\right)(i=1,2,3)$ and $b\left(q^{2}\right)$ are connected with the standard form factors $A_{i}\left(q^{2}\right)=(i=1,2,3)$ and $V\left(q^{2}\right)$ via the relations [1] 


$$
\begin{aligned}
A_{1}(0) & =\frac{1}{M_{D_{s}}+M_{\phi}} a_{1}(0)=0.43 \\
A_{2}(0) & =\left(M_{D_{s}}+M_{\phi}\right) a_{2}(0)=0.60 \\
A_{3}(0) & =\left(M_{D_{s}}+M_{\phi}\right) a_{3}(0)=-1.44 \\
V(0) & =\left(M_{D_{s}}+M_{\phi}\right) b(0)=0.81
\end{aligned}
$$

where $M_{D_{s}}=1.97 \mathrm{GeV}$ and $M_{\phi}=1.02 \mathrm{GeV}$ [10].

The theoretical values are in good agreement with the experimental data $[11,12]$

$$
\begin{array}{ll}
\left(R_{v}\right)_{\mathrm{th}}=\frac{V(0)_{\mathrm{th}}}{A_{1}(0)_{\mathrm{th}}}=1.9, & \left(R_{v}\right)_{\exp }=\left\{\begin{array}{l}
1.8 \pm 0.9 \pm 0.1[11] \\
1.4 \pm 0.5 \pm 0.3[12]
\end{array}\right. \\
\left(R_{2}\right)_{\mathrm{th}}=\frac{A_{2}(0)_{\mathrm{th}}}{A_{1}(0)_{\mathrm{th}}}=1.4, & \left(R_{2}\right)_{\exp }=\left\{\begin{array}{l}
1.1 \pm 0.6 \pm 0.1[11] \\
0.9 \pm 0.6 \pm 0.3[12]
\end{array}\right.
\end{array}
$$

\section{Conclusion}

By applying IMET supplemented by $(\mathrm{CHPT})_{q}$ we have evaluated the form factors of the $D_{s}^{+} \rightarrow \phi \mu^{+} \nu_{\mu}$ decay upto first order in current $s$-quark mass. The theoretical predictions compare reasonably well with experimental data. The proposed approach allows us to set up a relationship (10) between chiral corrections to the form factors of the decays $D_{s}^{+} \rightarrow \phi \mu^{+} \nu_{\mu}$ and $D \rightarrow$ $\bar{K}^{*} e^{+} \nu_{e}$. Unfortunately, the possibility of the experimental investigation of the relationship (10) goes beyond the available accuracy of present day experimental abilities.

\section{Acknowledgements}

ANI and NIT would like to thank Professor Abdus Salam, the International Atomic Energy Agency and UNESCO for hospitality at the International Centre for Theoretical Physics, Trieste. We acknowledge with pleasure fruitful discussions with Prof. G. E. Rutkovsky. 


\section{References}

[1] F. Hussain, A. N. Ivanov and N. I. Troitskaya, Phys. Lett. B 329 (1994) 98; ibid. B 334 (1994) E450.

[2] A. N. Ivanov, N. I. Troitskaya and M. Nagy, Phys. Lett. B 339 (1994) 167.

[3] F. Hussain, A. N. Ivanov and N. I. Troitskaya, Phys. Lett. B 348 (1995) 609.

[4] E. Eichten and F. L. Feinberg, Phys. Rev. D 23 (1981) 2724;

E. Eichten, Nucl. Phys. B 4 (Proc.Suppl.) (1988) 70;

M. B. Voloshin and M. A. Shifman, Sov. J. Nucl. Phys. 45 (1987) 292;

[5] H. D. Politzer and M. Wise, Phys. Lett. B 206 (1988) 681; ibid. B 208 (1988) 504.

[6] A. N. Ivanov, M. Nagy and N. I. Troitskaya, Int. J. Mod. Phys. A 7 (1992) 7305;

A. N. Ivanov, Int. J. Mod. Phys. A 8 (1993) 853;

A. N. Ivanov, N. I. Troitskaya and M. Nagy, Int. J. Mod. Phys.A 8 (1993) 2027; 3425;

A. N. Ivanov, N. I. Troitskaya and M. Nagy, Phys. Lett. B 308 (1993) 111

A. N. Ivanov and N. I. Troitskaya, " $\pi$ - and $a_{1}$ - meson physics in current algebra at the quark level", ICTP, Trieste, preprint IC/94/10, January 1994 (to appear in Nuovo Cimento A).

[7] Y. Nambu and G. Jona-Lasinio, Phys. Rev. 122 (1961) 345; ibid. 124 (1961) 246.

T. Eguchi, Phys. Rev. D 14 (1976) 2755; 
K. Kikkawa, Progr. Theor. Phys. 56 (1976) 947;

H. Kleinert, Proc. of Int. Summer School of Subnuclear Physics, Erice 1976, Ed. A. Zichichi, p.289.

[8] A. N. Ivanov and N. I. Troitskaya, Phys. Lett. B 342 (1995) 323.

[9] A. N. Ivanov and N. I. Troitskaya, Phys. Lett. B 345175.

[10] Particle Data Group, Phys. Rev. D 50 (1992) No.3, Part I.

[11] P. L. Fabertti et. al., Phys. Lett. B 328 (1994) 187.

[12] P. Avery et. al., CLEO Collaboration, Phys. Lett. B 337 (1994) 405. 